\title{
Necessary Conditions for the Global Rigidity of Direction-Length Frameworks
}

\author{
Bill Jackson • Peter Keevash
}

Received: 28 September 2009 / Revised: 16 January 2011 / Accepted: 17 January 2011 /

Published online: 29 January 2011

(C) Springer Science+Business Media, LLC 2011

\begin{abstract}
It is an intriguing open problem to give a combinatorial characterisation or polynomial algorithm for determining when a graph is globally rigid in $\mathbb{R}^{d}$. This means that any generic realisation is uniquely determined up to congruence when each edge represents a fixed length constraint. Hendrickson gave two natural necessary conditions, one involving connectivity and the other redundant rigidity. In general, these are not sufficient, but they do suffice in two dimensions, as shown by Jackson and Jordán. Our main result is an analogue of the redundant rigidity condition for frameworks that have both direction and length constraints. For any generic globally rigid direction-length framework in $\mathbb{R}^{d}$ with at least 2 length edges, we show that deleting any length edge results in a rigid framework. It seems harder to obtain a corresponding result when a direction edge is deleted: we can do this in two dimensions, under an additional hypothesis that we believe to be unnecessary. Our proofs use a lemma of independent interest, stating that a certain space parameterising equivalent frameworks is a smooth manifold. We prove this lemma using arguments from differential topology and the Tarski-Seidenberg theorem on semi-algebraic sets.
\end{abstract}

Keywords Rigidity · Global rigidity · Direction-length frameworks

\section{Introduction}

A finite configuration of points in Euclidean space with local constraints may be informally described as globally rigid if the constraints determine the point set

B. Jackson $(\varangle) \cdot$ P. Keevash

School of Mathematical Sciences, Queen Mary University of London, Mile End Road,

London E1 4NS, England, UK

e-mail: b.jackson@qmul.ac.uk

P. Keevash

e-mail: p.keevash@qmul.ac.uk 
up to congruence. This concept has applications in chemistry and for sensor networks, where one wants to deduce the global structure of a configuration from local measurements. A $d$-dimensional length framework $(G, p)$ consists of a graph $G=(V, E)$ and a map $p: V \rightarrow \mathbb{R}^{d}$. We say that $(G, q)$ and $(G, p)$ are lengthequivalent if $\|q(u)-q(v)\|=\|p(u)-p(v)\|$ for all $u v \in E$ and length-congruent if $\|q(u)-q(v)\|=\|p(u)-p(v)\|$ for all $u, v \in V$. Then we say that $(G, p)$ is globally length-rigid if any framework $(G, q)$ length-equivalent to $(G, p)$ is length-congruent to $(G, p)$. To exclude pathologies arising from algebraic relationships between the coordinates of vertices it is natural to consider generic maps $p$. A graph $G$ is globally length-rigid in $\mathbb{R}^{d}$ if $(G, p)$ is globally length-rigid for every generic $p: V \rightarrow \mathbb{R}^{d}$. A combination of results of Connelly [1] and Gortler, Healy and Thurston [2] gives an algebraic characterisation of global length-rigidity for generic frameworks and implies that it depends only on the structure of the underlying graph $G$. On the other hand, there is no known combinatorial characterisation or polynomial algorithm for determining when a graph $G$ is globally length-rigid in $\mathbb{R}^{d}$. It is easy to see that every complete graph is globally rigid in $\mathbb{R}^{d}$. Hendrickson [3] gave two necessary conditions for graphs $G$ which are not complete:

(i) $G$ must be $d$-connected, and

(ii) $G$ must be redundantly length-rigid in $\mathbb{R}^{d}$, i.e. $G \backslash e$ is length-rigid for any edge $e$ of $G$.

(Informally, a framework is length-rigid if it does not admit continuous motions other than to congruent frameworks, and a graph $G$ is length-rigid in $\mathbb{R}^{d}$ if $(G, p)$ is length-rigid for every generic $p: V \rightarrow \mathbb{R}^{d}$.) For general $d$, these conditions are not sufficient for global length-rigidity, as shown by Connelly [1]. They do suffice for $d=2$, as shown by Jackson and Jordán [4], who proved that a 2-dimensional generic length framework $(G, p)$ is globally length-rigid if and only if either $G$ is a complete graph on at most 3 vertices, or $G$ is 3 -connected and redundantly rigid.

In this paper, we consider frameworks in which the local constraints can involve both lengths and directions. We consider mixed graphs $G=(V ; D, L)$, where $D$ and $L$ are edge sets representing direction and length constraints. Given maps $p, q$ from $V$ to $\mathbb{R}^{d}$ we say that the direction-length frameworks $(G, p)$ and $(G, q)$ are equivalent if $q(u)-q(v)$ is a scalar multiple of $p(u)-p(v)$ for all $u v \in D$ with $p(u) \neq p(v)$, and $\|q(u)-q(v)\|=\|p(u)-p(v)\|$ for all $u v \in L$. They are congruent if $(G, q)$ can be obtained from $(G, p)$ by a translation and a dilation by \pm 1 . Similarly to before, we say that $(G, p)$ is globally rigid if any framework $(G, q)$ equivalent to $(G, p)$ is congruent to $(G, p)$, and is rigid if every continuous motion of $(G, p)$ results in a congruent framework. These concepts are illustrated in Fig. 1. We will investigate when $G$ is globally rigid, i.e. $(G, p)$ is globally rigid for any generic map $p$.

Suppose $(G, p)$ is a $d$-dimensional generic direction-length framework. It is certainly necessary for $G$ to be connected if $(G, p)$ is to be rigid, let alone globally rigid. Also, 2-connectivity is necessary for global rigidity, as if $x$ is a cutvertex of $G$ then we can obtain a realisation $\left(G, p^{\prime}\right)$ that is equivalent but not congruent to $(G, p)$ by inverting one component of $G \backslash x$ about the point $p(x)$, without changing the rest of the realisation. On the other hand, if $G=(V ; D, L)$ is 2-connected and $D=L$ then $(G, p)$ is globally rigid if and only if $G$ is 2-connected, see [5, Theorem 7.2], 

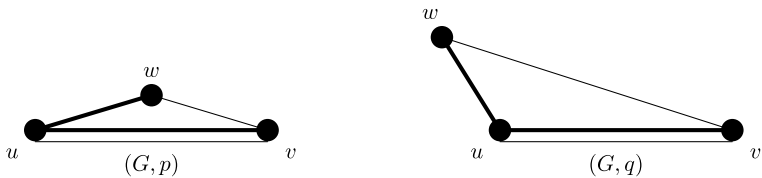

Fig. 1 Two equivalent but non-congruent frameworks. We use thick or thin lines to indicate edges representing length or direction constraints, respectively. The frameworks are rigid but not globally rigid

so 3-connectivity is no longer necessary for global rigidity in $\mathbb{R}^{d}$. However, an analogue of Hendrickson's connectivity condition may be obtained by considering more restricted cuts: if $(G, p)$ is globally rigid then there can be no cutset $X \subseteq V$ of size at most $d$ such that there is a component $C$ of $G \backslash X$ that contains only length edges, as then we could obtain a realisation $\left(G, p^{\prime}\right)$ which is equivalent but not congruent to $(G, p)$ by reflecting $C$ in a hyperplane containing the points $p(x), x \in X$. The main result of [5] is that this connectivity condition is both necessary and sufficient for the global rigidity of redundantly rigid 2-dimensional generic direction-length frameworks when $|D \cup L|=2|V|-1$, i.e. $D \cup L$ is a circuit in the corresponding rigidity matroid.

Now we turn to an analogue of Hendrickson's redundant rigidity condition. Our main theorem achieves this when a length edge is deleted.

Theorem 1.1 (Length-redundant rigidity) Suppose that $(G, p)$ is a generic globally rigid direction-length framework in $\mathbb{R}^{d}$ with at least 2 length edges. Then $G \backslash e$ is rigid in $\mathbb{R}^{d}$ for any length edge e of $G$.

The proof uses the following lemma of independent interest, established using arguments from differential topology and the Tarski-Seidenberg theorem on semialgebraic sets. (The framework space parameterises frameworks equivalent to $(G, p)$ and will be formally defined in the next section, as will the rank of $(G, p)$.)

Lemma 1.2 (Manifold Lemma) Suppose $(G, p)$ is a generic direction-length framework in $\mathbb{R}^{d}$ with rank $d|V|-d-t$, for some $t \geq 1$, and $v_{0} \in V$. Then the framework space $S_{G, p, v_{0}}$ is a smooth manifold of dimension $t$.

It is also natural to consider the result of deleting a direction edge, rather than a length edge, from a $d$-dimensional generic globally rigid direction-length framework. This can reduce the rank of the rigidity matrix by up to $d-1$, so we expect a more complicated behaviour for $d \geq 3$. However, in the special case $d=2$, we can obtain the following weakening of Hendrickson's redundant rigidity condition.

Theorem 1.3 Suppose $(G, p)$ is a 2-dimensional generic globally rigid directionlength framework, $e$ is a direction edge of $G$ and $G \backslash e$ has a rigid subgraph with more than one vertex. Then $G \backslash e$ is either rigid or unbounded in $\mathbb{R}^{2}$. 


\section{Definitions}

Our graphs will not have loops but may have parallel edges. A mixed graph $G=$ $(V ; D, L)$ consists of a graph $G$ on a vertex set $V$ in which the edge set $E$ is partitioned into two parts $D$ and $L$. We refer to edges in $D$ as direction edges and edges in $L$ as length edges.

A $d$-dimensional direction-length framework is a pair $(G, p)$, where $G=$ $(V ; D, L)$ is a mixed graph and $p$ is a map from $V$ to $\mathbb{R}^{d}$. We say that $(G, p)$ is a direction-length realisation of $G$ in $\mathbb{R}^{d}$. We will identify $p$ with the vector in $\mathbb{R}^{d|V|}$ obtained by concatenating the vectors $p(v)$ for $v \in V$ (in some fixed order). Given a fixed vertex $v_{0} \in V$, we say that $p$ is in standard position (with respect to $v_{0}$ ) if $p\left(v_{0}\right)=0 \in \mathbb{R}^{d}$. In this case, we will identify $p$ with the vector in $\mathbb{R}^{d|V|-d}$ obtained by concatenating the vectors $p(v)$ for $v \in V \backslash\left\{v_{0}\right\}$. Also, we identify any function $f$ defined on the set of standard position realisations with a function on $\mathbb{R}^{d|V|-d}$, and also with a function on $\left\{p \in \mathbb{R}^{d|V|}: p\left(v_{0}\right)=0\right\}$.

Two direction-length frameworks $(G, p)$ and $(G, q)$ are equivalent if $q(u)-q(v)$ is a scalar multiple of $p(u)-p(v)$ for all $u v \in D$ with $p(u) \neq p(v)$, and $\| p(u)-$ $p(v)\|=\| q(u)-q(v) \|$ for all $u v \in L$, where $\|\cdot\|$ denotes the Euclidean norm in $\mathbb{R}^{d}$. They are congruent if there exists a $\lambda \in\{1,-1\}$ such that $p(u)-p(v)=\lambda(q(u)-$ $q(v))$ for all $u, v \in V$, i.e. $(G, q)$ can be obtained from $(G, p)$ by a translation and a dilation by \pm 1 .

The direction-length framework $(G, p)$ is globally rigid if every framework which is equivalent to $(G, p)$ is congruent to $(G, p)$.

The framework space $S_{G, p, v_{0}} \subseteq \mathbb{R}^{d|V|-d}$ consists of all $q$ in standard position with respect to $v_{0}$ with $(G, q)$ equivalent to $(G, p)$. We claim that $S_{G, p, v_{0}}$ is a closed set. To see this, consider any convergent sequence $q_{1}, q_{2}, \ldots$ in $S_{G, p, v_{0}}$ and let $q=\lim _{i \rightarrow \infty} q_{i}$. For each $u v \in D$ we have $\left[q_{i}(u)-q_{i}(v)\right] \cdot[p(u)-p(v)]=0$, and hence $[q(u)-q(v)] \cdot[p(u)-p(v)]=0$. Similarly, for each $u v \in L$ we have $\left\|q_{i}(u)-q_{i}(v)\right\|=\|p(u)-p(v)\|$ and hence $\|q(u)-q(v)\|=\|p(u)-p(v)\|$. Thus $q \in S_{G, p, v_{0}}$, so $S_{G, p, v_{0}}$ is closed.

We say $(G, p)$ is rigid if there exists an $\varepsilon>0$ such that if a framework $(G, q)$ is equivalent to $(G, p)$ and satisfies $\|p(v)-q(v)\|<\varepsilon$ for all $v \in V$ then $(G, q)$ is congruent to $(G, p)$. Equivalently, every continuous motion of the points $p(v)$, $v \in V$ respecting the length and direction constraints results in a framework which is congruent to $(G, p)$.

A direction-length framework $(G, p)$ is bounded if there exists a real number $K$ such that $\|q(u)-q(v)\|<K$ for all $u, v \in V$ whenever $(G, q)$ is a framework equivalent to $(G, p)$.

A point $v \in \mathbb{R}^{d}$ is generic if its coordinates are algebraically independent over the rationals. A direction-length framework $(G, p)$ is generic if the set containing the coordinates of all of the vertices is algebraically independent over the rationals. We say $(G, p)$ is quasi-generic if it is congruent to a generic configuration. Note that if $(G, p)$ is generic and $v_{0} \in V$ then the realisation $\left(G, p_{0}\right)$ defined by $p_{0}(v)=$ $p(v)-p\left(v_{0}\right)$ is quasi-generic and in standard position.

We say that a property $\mathrm{P}$ of frameworks is generic if whenever some generic realisation of a graph $G$ has property $\mathrm{P}$ then all generic realisations of $G$ have property $\mathrm{P}$. 
If $\mathrm{P}$ is a generic property then we say that a graph $G$ has property $\mathrm{P}$ if some generic realisation of $G$ has property $\mathrm{P}$ (or equivalently, all generic realisations of $G$ have property $\mathrm{P}$ ).

Given a realisation $p$ of $G$ and a direction edge $e=u v$ we let $B_{e}$ be a $(d-1) \times d$ matrix whose rows are a basis for the subspace of $\mathbb{R}^{d}$ orthogonal to $\langle p(u)-p(v)\rangle$ if $p(u) \neq p(v)$, and $B_{e}=0$ otherwise. A rigidity matrix for $(G, p)$ is a $((d-1)|D|+$ $|L|) \times d|V|$ matrix $R(G, p)$ constructed as follows. We first choose an arbitrary reference orientation for the edges of $D$, and use the notation $e=u v$ to mean that $e$ has been oriented from $u$ to $v$. Each edge in $D$ corresponds to $d-1$ consecutive rows of $R(G, p)$, each edge in $L$ to one row of $R(G, p)$, and each vertex in $V$ to $d$ consecutive columns of $R(G, p)$. The submatrix of $R(G, p)$ with rows labelled by $e=u v \in D$ and columns labelled by $x \in V$ is $B_{e}$ if $x=u$, is $-B_{e}$ if $x=v$, and is the $(d-1) \times d$ zero matrix otherwise. The submatrix of $B(G, p)$ with row labelled by $e=u v \in L$ and columns labelled by $x \in V$ is $p(u)-p(v)$ if $x=u$, is $p(v)-p(u)$ if $x=v$, and is zero otherwise.

Let $Z(G, p)$ be the null space of $R(G, p)$. We refer to vectors in $Z(G, p)$ as infinitesimal motions of $(G, p)$. The labelling of the columns of $R(G, p)$ allows us to consider each infinitesimal motion $z$ as a map from $V$ to $\mathbb{R}^{d}$ with the properties that $B_{u v}(z(u)-z(v))=0$ for all $e=u v \in D$ and $(p(u)-p(v)) \cdot(z(u)-z(v))=0$ for all $u v \in L$. For $e=u v \in D$ the condition $B_{e}(z(u)-z(v))=0$ is equivalent to $z(u)-z(v)$ being parallel to $p(u)-p(v)$, so $Z(G, p)$ depends only on the framework $(G, p)$ : it is independent of the choice of the bases $B_{e}, e \in D$. In particular, $\operatorname{dim} Z(G, p)$ and hence rank $R(G, p)$ depend only on the framework $(G, p)$. We will refer to rank $R(G, p)$ as the rank of $(G, p)$.

Given a realisation $(G, p)$ of $G$, we say that an edge $e=u v \in D$ is vertical in $(G, p)$ if the last coordinate of $p(u)-p(v)$ is zero. When $(G, p)$ has no vertical direction edges, it will be helpful to refer to a particular rigidity matrix which we call the standard rigidity matrix. This is defined as follows: for each $e=u v \in D$ and $p(u)-p(v)=\left(a_{1}, \ldots, a_{d}\right)$, we take the rows of $B_{e}$ as the vectors $b^{1}, \ldots, b^{d-1}$, where $b^{i}$ is equal to $a_{d}$ in coordinate $i$, to $-a_{i}$ in coordinate $d$, and 0 in the other coordinates.

It will also be useful to construct the standard rigidity matrix via the Jacobian matrix of the following map. For $x=\left(x_{1}, x_{2}, \ldots, x_{d}\right) \in \mathbb{R}^{d}$, let $l(x)=\|x\|^{2}$, and when $x_{d} \neq 0$ let $t(x)=\left(x_{1} / x_{d}, x_{2} / x_{d}, \ldots, x_{d-1} / x_{d}\right)$. Let $G=(V ; D, L)$ be a graph with $D \cup L=\left\{e_{1}, e_{2}, \ldots, e_{m}\right\}$. Choose a reference orientation for the edges of $D \cup L$. Let $T$ be the set of all points $p \in \mathbb{R}^{d|V|}$ such that $(G, p)$ has no vertical direction edges. For each $p \in T$ and $e_{i}=u v \in D \cup L$ let $f_{i}(p)=t(p(u)-p(v))$ if $e_{i} \in$ $D$, and $f_{i}(p)=l(p(u)-p(v))$ if $e_{i} \in L$. The rigidity map $f_{G}: T \rightarrow \mathbb{R}^{(d-1)|D|+|L|}$ is defined by putting $f_{G}(p)=\left(f_{1}(p), f_{2}(p), \ldots, f_{m}(p)\right)$. Now consider any edge $e_{i}=u v$ and write $p(u)=\left(a_{1}, \ldots, a_{d}\right), p(v)=\left(b_{1}, \ldots, b_{d}\right)$. If $e_{i} \in L$ then $f_{i}(p)=$ $\sum_{j=1}^{d}\left(a_{j}-b_{j}\right)^{2}$, so for $1 \leq j \leq d$ we have $\frac{\partial f_{i}(p)}{\partial a_{j}}=2\left(a_{j}-b_{j}\right)$ and $\frac{\partial f_{i}(p)}{\partial b_{j}}=-2\left(a_{j}-\right.$ $b_{j}$ ). (Derivatives with respect to variables not appearing in $p(u)$ or $p(v)$ are of course zero.) Thus the row in the Jacobian corresponding to $e_{i}$ is obtained by multiplying that of the standard rigidity matrix by 2 , which does not affect the rank. Next suppose that $e_{i} \in D$. Then $f_{i}(p) \in \mathbb{R}^{d-1}$ has $j$ th coordinate $f_{i}(p)_{j}=\frac{a_{j}-b_{j}}{a_{d}-b_{d}}$. We have $\frac{\partial f_{i}(p)_{j}}{\partial a_{j}}=$ $\frac{1}{a_{d}-b_{d}}$ and $\frac{\partial f_{i}(p)_{j}}{\partial a_{d}}=-\frac{a_{j}-b_{j}}{\left(a_{d}-b_{d}\right)^{2}}$. Multiplying these rows through by $\left(a_{d}-b_{d}\right)^{2}$ we 
obtain the same basis of the space orthogonal to $p(u)-p(v)$ that is used in the standard rigidity matrix.

Note that $f_{G}(q)=f_{G}(p)$ for those $q \in S_{G, p, v_{0}}$ such that $f_{G}(q)$ is defined. However, there may be some points $q \in S_{G, p, v_{0}}$ such that $f_{G}(q)$ is undefined: this occurs when the endpoints of some direction edge coincide in $q$.

For any $a \in \mathbb{R}^{d}$ the translation given by $z(v)=a$ for all $v \in V$ is an infinitesimal motion, so $\operatorname{dim} Z(G, p) \geq d$ and $\operatorname{rank} R(G, p) \leq d|V|-d$. We can 'factor out' translations by restricting attention to realisations $p$ that are in standard position, i.e. satisfy $p\left(v_{0}\right)=0$ for some fixed $v_{0} \in V$. Write $R(G, p)_{v_{0}}$ for the matrix obtained from $R(G, p)$ by deleting the $d$ columns corresponding to $v_{0}$ and let $Z(G, p)_{v_{0}}$ be its null space. Since all translations belong to $Z(G, p) \backslash Z(G, p)_{v_{0}}$ we have $\operatorname{dim} Z(G, p)_{v_{0}}=\operatorname{dim} Z(G, p)-d$, so rank $R(G, p)_{v_{0}}=d|V|-d-\operatorname{dim} Z(G, p)_{v_{0}}=$ $d|V|-\operatorname{dim} Z(G, p)=\operatorname{rank} R(G, p)$. We say that the framework $(G, p)$ is infinitesimally rigid if rank $R(G, p)=d|V|-d$, and is independent if the rows of $R(G, p)$ are linearly independent. Infinitesimal rigidity and independence are both generic properties of graphs, as the rank of $R(G, p)$ is the same for all generic realisations of $G$. We denote the rank of the rigidity matrix of a generic realisation of $G$ in $\mathbb{R}^{d}$ by $r_{d}(G)$. Then $G$ is independent in $\mathbb{R}^{d}$ if $r_{d}(G)=(d-1)|D|+|L|$ and infinitesimally rigid if $r_{d}(G)=d|V|-d$. We refer the reader to [11] for further details of the 2-dimensional case.

It is shown in [7] that a generic framework $(G, p)$ is rigid if and only if it is infinitesimally rigid, and is bounded if and only if the augmented framework $\left(G^{+}, p\right)$ is infinitesimally rigid. $\left(\left(G^{+}, p\right)\right.$ is obtained from $(G, p)$ by adding a direction edge between all pairs of vertices of $G$ which are joined by a length edge.) It follows that rigidity and boundedness are both generic properties of direction-length frameworks. It is not known whether global rigidity is also a generic property.

\section{Differential Topology Preliminaries}

In this section, we recall some basic concepts and facts of differential topology. We refer the reader to [9] for an introduction to this subject. Let $X$ be a smooth manifold, $f: X \rightarrow \mathbb{R}^{n}$ be a smooth map, and $k$ be the maximum rank of its derivative $\left.d f\right|_{y}$ over all $y \in X$. A point $x \in X$ is a regular point of $f$ if rank $\left.d f\right|_{x}=k$, otherwise $x$ is a critical point. A point $y \in f(X)$ is a critical value if $y=f(x)$ for some critical point $x$, otherwise $y$ is a regular value. A fundamental theorem of Sard states that the set of critical values of $f$ has measure zero (see [9, Chap. 3]).

Theorem 3.1 (Sard) Let $f: U \rightarrow \mathbb{R}^{n}$ be a smooth map defined on an open subset $U$ of $\mathbb{R}^{m}$ and $C$ be the set of critical points of $f$. Then $f(C)$ has Lebesgue measure zero in $\mathbb{R}^{n}$.

We also need the fact that if $y$ is a regular value then $f^{-1}(y)$ is an $(m-k)$ dimensional manifold (see [9, p. 11, Lemma 1]).

Lemma 3.2 Let $X$ be a smooth manifold of dimension $m$ and $f: X \rightarrow \mathbb{R}^{n}$ be a smooth map. Suppose that $x \in X$ and that $f(x)$ is a regular value of $f$ with rank $\left.d f\right|_{x}=k$. Then $f^{-1}(f(x))$ is an $(m-k)$-dimensional smooth manifold. 
Next we recall the Inverse Function Theorem: if $U$ is open in $\mathbb{R}^{k}, f: U \rightarrow \mathbb{R}^{k}$ is smooth and the derivative $\left.d f\right|_{x}: \mathbb{R}^{k} \rightarrow \mathbb{R}^{k}$ is non-singular, then $f$ maps any sufficiently small open neighbourhood $U^{\prime}$ of $x$ diffeomorphically onto an open set $f\left(U^{\prime}\right)$. The following lemma is a simple consequence of this.

Lemma 3.3 Let $U$ be an open subset of $\mathbb{R}^{m}, f: U \rightarrow \mathbb{R}^{n}$ be a smooth map and $x \in U$ be a regular point of $f$. Suppose that the rank of $\left.d f\right|_{x}$ is $n$. Then there exists an open neighbourhood $W \subseteq U$ of $x$ such that $f(W)$ is an open neighbourhood of $f(x)$ in $\mathbb{R}^{n}$.

Proof Choose a linear map $L: U \rightarrow \mathbb{R}^{m-n}$ such that $L$ is injective on the null space of $\left.d f\right|_{x}$ and define $F: U \rightarrow \mathbb{R}^{m}$ by $F(y)=(f(y), L(y))$. Then $\left.\operatorname{rank} d F\right|_{x}=m$, so by the inverse function theorem, there exists an open neighbourhood $W$ of $x$ such that $F(W)=f(W) \times L(W)$ is diffeomorphic to $W$. Hence $f(W) \times L(W)$ is an open subset of $\mathbb{R}^{m}$ and so $f(W)$ is an open subset of $\mathbb{R}^{n}$.

Next we need a lemma for computing the rank of the derivative when a function is restricted to a submanifold.

Lemma 3.4 Let $M$ be a smooth manifold and $x \in M$. Suppose $\theta: M \rightarrow \mathbb{R}^{a}$ and $F: M \rightarrow \mathbb{R}^{b}$ are smooth maps, and define $H: M \rightarrow \mathbb{R}^{a+b}$ by $H(y)=(\theta(y), F(y))$. Suppose $\theta(x)$ is a regular value of $\theta$, let $X$ be the submanifold $\theta^{-1}(\theta(x))$ of $M$, and let $f$ be the restriction of $F$ to $X$. Then $\left.\operatorname{rank} d f\right|_{x}=\left.\operatorname{rank} d H\right|_{x}-\left.\operatorname{rank} d \theta\right|_{x}$.

Proof Let $S$ be the tangent space of $M$ at $x$ and $T$ the tangent space of $X$ at $x$. Then $T=\left\{y \in S:\left.d \theta\right|_{x}(y)=0\right\}$ and $\operatorname{dim} T=\operatorname{dim} S-\left.\operatorname{rank} d \theta\right|_{x}$ by Lemma 3.2. Also, the null spaces of $\left.d f\right|_{x}$ and $\left.d H\right|_{x}$ are equal, as

$$
\begin{aligned}
Z\left(\left.d f\right|_{x}\right) & =\left\{y \in T:\left.d f\right|_{x}(y)=0\right\}=\left\{y \in S:\left.d F\right|_{x}(y)=\left.d \theta\right|_{x}(y)=0\right\} \\
& =\left\{y \in S:\left.d H\right|_{x}(y)=0\right\}=Z\left(\left.d H\right|_{x}\right) .
\end{aligned}
$$

Therefore,

$$
\begin{aligned}
\left.\operatorname{rank} d f\right|_{x} & =\operatorname{dim} T-\operatorname{dim} Z\left(\left.d f\right|_{x}\right)=\operatorname{dim} S-\left.\operatorname{rank} d \theta\right|_{x}-\operatorname{dim} Z\left(\left.d H\right|_{x}\right) \\
& =\left.\operatorname{rank} d H\right|_{x}-\left.\operatorname{rank} d \theta\right|_{x} .
\end{aligned}
$$

An important corollary of the previous lemma is that of restriction to a fixed subspace.

Lemma 3.5 Let $A$ be an $m \times n$ real matrix and $X=\left\{y \in \mathbb{R}^{n}: A y=0\right\}$. Suppose $F: \mathbb{R}^{n} \rightarrow \mathbb{R}^{t}$ is a smooth map and let $f$ be the restriction of $F$ to $X$. Let $x \in X$ and let $B$ be the Jacobian of $F$ evaluated at $x$. Then $\left.\operatorname{rank} d f\right|_{x}=\operatorname{rank}\left(\begin{array}{l}A \\ B\end{array}\right)-\operatorname{rank} A$.

Proof Apply Lemma 3.4 with $M=\mathbb{R}^{n}$ and $\theta: \mathbb{R}^{n} \rightarrow \mathbb{R}^{m}$ defined by $\theta(y)=A y$.

Finally, we need a result showing that the quotient of a smooth manifold by a group action is again a smooth manifold, under appropriate conditions. Suppose that a finite 
group $G$ acts on a smooth manifold $M$. The action is smooth if $x \mapsto g x$ is a smooth map from $M$ to $M$ for every $g \in G$. The action is free if $g x \neq x$ for every $g \neq 1$ and every $x \in M$. The following statement is well-known, and can be easily deduced, e.g. from [8, Theorem 9.19]. (See [8, Chap. 9] for the definition of the smooth structure on $M / G$ and further details.)

Theorem 3.6 Suppose a finite group $G$ acts smoothly and freely on a smooth manifold $M$. Then the quotient space $M / G$ is a smooth manifold.

\section{Redundant Rigidity}

In this section, we will assume the manifold lemma (Lemma 1.2) and prove our theorems on redundant rigidity. We first illustrate the proof technique by considering the framework $(G, p)$ of Fig. 1 . We can show that $(G, p)$ is not globally rigid by constructing the equivalent but non-congruent framework $(G, q)$ in two different ways. We first delete the length edge $e=u v$. The resulting framework $(G \backslash e, p)$ is not rigid. We may move it continuously keeping the vertex $u$ fixed, rotating $w$ around the circle through $p(w)$ which is centred on $p(u)$, and moving $v$ along the line through $p(u)$ and $p(v)$. We will eventually reach the framework $(G \backslash e, q)$ which is equivalent to $(G \backslash e, p)$ and also satisfies $\|q(u)-q(v)\|=\|p(u)-p(v)\|$. This is essentially the proof technique of Theorem 1.1. Alternatively, we can delete the direction edge $f=w v$. Then $(G \backslash f, p)$ is not rigid. We may move it continuously keeping the vertices $u$ and $v$ fixed and rotating $w$ around the circle through $p(w)$ which is centred on $p(u)$. We will eventually reach the framework $(G \backslash f, q)$ which is equivalent to $(G \backslash f, p)$ and in which $q(w)-q(v)$ is a scalar multiple of $p(w)-p(v)$. This is essentially the proof technique of Theorem 1.3.

We will need the following result, which is Lemma 8.2 in [7], to prove Theorem 1.1 .

Lemma 4.1 Suppose that $(G, p)$ is a generic globally rigid direction-length framework in $\mathbb{R}^{d}$ with at least two length edges and $e$ is a length edge of $G$. Then $G \backslash e$ is bounded.

Proof of Theorem 1.1 Suppose for a contradiction that $H=G \backslash e$ is not rigid. We have $r_{d}(G)=d|V|-d$ as $G$ is rigid, $r_{d}(H)<r_{d}(G)$ as $H$ is not rigid, so $r_{d}(H)=$ $d|V|-d-1$, as the rigidity matrix $R(H, p)$ is obtained from $R(G, p)$ by deleting a single row corresponding to the length edge $e$. Fix $v_{0} \in V$ and let $p_{0}$ be the realisation obtained from $p$ by translating $v_{0}$ to the origin, i.e. $p_{0}(v)=p(v)-p\left(v_{0}\right)$ for all $v \in V$. Then $p_{0}$ is quasi-generic and we can identify $p_{0}$ with a generic vector in $\mathbb{R}^{d|V|-d}$. By the Manifold Lemma (Lemma 1.2), the framework space $S_{H, p, v_{0}}$ is a smooth 1-dimensional manifold. Also, $S_{H, p, v_{0}}$ is bounded, since $H$ is bounded by Lemma 4.1, and we observed in Sect. 2 that it is closed.

Next note that $-q \in S_{H, p, v_{0}}$ for every $q \in S_{H, p, v_{0}}$. Also, $0 \notin S_{H, p, v_{0}}$, as $H$ has at least one length edge. Then we can identify $q$ with $-q$ for all $q \in S_{H, p, v_{0}}$ to obtain a smooth 1-dimensional manifold $X=S_{H, p, v_{0}} /\{-1,1\}$ that is closed and 
bounded. This last statement follows from that the fact that the action of the group $\{-1,1\}$ by multiplication is smooth and free (see Theorem 3.6). Let $C$ be the connected component of $X$ containing $p_{0}$. Then $C$ is diffeomorphic to a circle by the classification of 1-manifolds (see the appendix to [9]). Write $e=x y$ and let $M=\left(\mathbb{R}^{d|V|-d} \backslash\{0\}\right) /\{-1,1\}$, which is a smooth manifold by the same argument as for $X$. We will abuse notation and let $q \in \mathbb{R}^{d|V|-d} \backslash\{0\}$ also denote its equivalence class $\{q,-q\}$ in $M$. Then $F: \mathbb{R}^{d|V|-d} \rightarrow \mathbb{R}$ given by $F(q)=\|q(x)-q(y)\|^{2}$ is welldefined. Let $f$ be restriction of $F$ to $C$. We can view the rigidity maps $f_{G}$ and $f_{H}$ as being defined on $M$. Note that the rigidity map $f_{G}$ is obtained from $f_{H}$ by adding a coordinate corresponding to $F$.

Since $p$ is generic, $f_{H}(p)$ is a regular value of $f_{H}$. Applying Lemma 3.4 with $\theta=f_{H}$ and $H=(\theta, F)=f_{G}$, for $q \in C$ we can calculate rank $\left.d f\right|_{q}=\left.\operatorname{rank} d H\right|_{q}-$ $\left.\operatorname{rank} d \theta\right|_{q}=R(G, q)-R(H, q)$. When $q=p_{0}$ we have $R\left(G, p_{0}\right)=r_{d}(G)$ and $R\left(H, p_{0}\right)=r_{d}(H)<r_{d}(G)$, so rank $\left.d f\right|_{p_{0}}=1$. It follows that $f\left(p_{0}\right)$ is not an extremal value of $f(q)$ for $q \in C$, i.e. we can find $q_{1}$ and $q_{2}$ in a neighbourhood of $p_{0}$ in $C$ with $f\left(q_{1}\right)<f\left(p_{0}\right)<f\left(q_{2}\right)$. Since there are two paths in $C$ joining $q_{1}$ and $q_{2}$, by the intermediate value theorem we can find $q \in C, q \neq p_{0}$ with $f(q)=f\left(p_{0}\right)$. Then $(G, q)$ is equivalent to but not congruent to $(G, p)$, contradicting our assumption that $G$ is globally rigid. We deduce that $H=G \backslash e$ is rigid.

For Theorem 1.3, we will need the following lemma, which is Lemma 3.4 in [6].

Lemma 4.2 Suppose $G$ is a rigid mixed graph, $p$ is a quasi-generic 2-dimensional realisation of $G$ and $(G, q)$ is equivalent to $(G, p)$. Then $q(u) \neq q(v)$ for all $u v \in D$.

Proof of Theorem 1.3 Suppose $H=G \backslash e$ has a rigid subgraph $H_{0}$ with $\left|V\left(H_{0}\right)\right| \geq 2$, and suppose for a contradiction that $H$ is bounded but not rigid. Fix $v_{0} \in V\left(H_{0}\right)$ and let $p_{0}$ be the realisation obtained from $p$ by translating $v_{0}$ to the origin. Similarly to the proof of Theorem 1.1, the connected component $C$ of $S_{H, p, v_{0}}$ containing $p_{0}$ is diffeomorphic to a circle (here we do not quotient out by $\{-1,1\}$ ). Also, we claim that $-p_{0} \notin C$. To see this, fix $v_{1} \in V\left(H_{0}\right)$ with $v_{1} \neq v_{0}$. Since $H_{0}$ is rigid and $q\left(v_{0}\right)=0$ for all $q \in C$ we have $q\left(v_{1}\right)=p_{0}\left(v_{1}\right)$ for all $q \in C$. (This is because $\left\{q \in C: q\left(v_{1}\right)=\right.$ $\left.p_{0}\left(v_{1}\right)\right\}$ is an open subset of $C$ by definition of rigidity, and it is clearly closed, so must be all of $C$.) Since $p_{0}$ is a realisation we have $p_{0}\left(v_{1}\right) \neq p_{0}\left(v_{0}\right)=0$, so $p_{0}\left(v_{1}\right) \neq$ $-p_{0}\left(v_{1}\right)$, and we deduce that $-p_{0} \notin C$.

Write $e=x y$, and for $q \in \mathbb{R}^{2|V|-2}$ with $q(x) \neq q(y)$ define $F(q)=\frac{q(x)-q(y)}{\|q(x)-q(y)\|}$. Then $F$ is a smooth map defined on an open subset of $\mathbb{R}^{2|V|-2}$ taking values in the unit circle $C^{\prime} \subseteq \mathbb{R}^{2}$. Also, $G$ is rigid (since it is globally rigid) and any realisation $q$ of $H$ with $q(x)=q(y)$ would also be a realisation of $G$ with $q(x)=q(y)$, which is impossible by Lemma 4.2. Therefore, $F$ is defined at all points of $C$ and we can let $f$ be the restriction of $F$ to $C$. For any connected open subset $U \subseteq C^{\prime}$ not containing $(-1,0)$ or $(1,0)$, we can define $t: U \rightarrow \mathbb{R}$ by $t\left(a_{1}, a_{2}\right)=a_{1} / a_{2}$. Note that $t$ is a diffeomorphism between $U$ and an open interval $t(U) \subseteq \mathbb{R}$. For $q \in U$ the rigidity map $f_{G}$ is obtained from $f_{H}$ by adding a row corresponding to $t \circ F$. Applying Lemma 3.4 with $\theta=f_{H}$ and $H=(\theta, t \circ F)=f_{G}$, for $q \in C$ we can calculate $\left.\operatorname{rank} d(t \circ f)\right|_{q}=\left.\operatorname{rank} d H\right|_{q}-\left.\operatorname{rank} d \theta\right|_{q}=R(G, q)-R(H, q)$. 
Since $p$ is generic we can choose $U$ to contain $p_{0}$. We have $R\left(G, p_{0}\right)=r_{d}(G)$ and $R\left(H, p_{0}\right)=r_{d}(H)<r_{d}(G)$, so rank $\left.d(t \circ f)\right|_{p_{0}}=1$. It follows that $(t \circ f)\left(p_{0}\right)$ is not a critical value of $t \circ f$, and so $f\left(p_{0}\right)$ is not a critical value of $f$.

To finish the proof, we show that we can find $q \in C, q \neq p_{0}$ with $f(q)$ equal to $f\left(p_{0}\right)$ or $-f\left(p_{0}\right)$. For suppose that $-f\left(p_{0}\right) \notin f(C)$. Let $s: C^{\prime} \backslash\left\{-f\left(p_{0}\right)\right\} \rightarrow \mathbb{R}$ be a diffeomorphism, e.g. stereographic projection. Then $s \circ f$ is a smooth map from $C$ to $\mathbb{R}$ and $(s \circ f)\left(p_{0}\right)$ is a regular value of $s \circ f$. Thus we can find $q_{1}$ and $q_{2}$ in a neighbourhood of $p_{0}$ in $C$ with $(s \circ f)\left(q_{1}\right)<(s \circ f)\left(p_{0}\right)<(s \circ f)\left(q_{2}\right)$. Since there are two paths in $C$ joining $q_{1}$ and $q_{2}$, by the intermediate value theorem we can find another realisation $q \in C, q \neq p_{0}$ with $(s \circ f)(q)=(s \circ f)\left(p_{0}\right)$, i.e. $f(q)=f\left(p_{0}\right)$.

We have shown that there is $q \in C, q \neq p$ with $f(q)$ equal to $f\left(p_{0}\right)$ or $-f\left(p_{0}\right)$. Then $(G, q)$ is equivalent to but not congruent to $(G, p)$, contradicting our assumption that $G$ is globally rigid. We deduce that $H=G \backslash e$ is rigid.

\section{Proof of the Manifold Lemma}

In this section, we will prove the Manifold Lemma (Lemma 1.2). We will require the Tarski-Seidenberg decision procedure, which can be formulated in terms of semialgebraic sets as follows. A subset $S$ of $\mathbb{R}^{n}$ is semi-algebraic over $\mathbb{Q}$ if it can be expressed as a finite union of sets of the form

$$
\left\{x \in \mathbb{R}^{n}: P_{i}(x)=0,1 \leq i \leq s \text { and } Q_{j}(x)>0,1 \leq j \leq t\right\},
$$

where $P_{i} \in \mathbb{Q}\left[X_{1}, \ldots, X_{n}\right]$ for $1 \leq i \leq s$, and $Q_{j} \in \mathbb{Q}\left[X_{1}, \ldots, X_{n}\right]$ for $1 \leq j \leq t .1$

Theorem 5.1 (Tarski-Seidenberg [12]) Let $S \subseteq \mathbb{R}^{n+k}$ be semi-algebraic over $\mathbb{Q}$ and $\pi: \mathbb{R}^{n+k} \rightarrow \mathbb{R}^{n}$ be the projection onto the first $n$ coordinates. Then $\pi(S)$ is semialgebraic over $\mathbb{Q}$.

Given a quasi-generic realisation $(G, p)$ of a mixed graph $G=(V ; D, L)$ in $\mathbb{R}^{d}$ we use $R(G, p)$ to denote that the standard rigidity matrix of $G$. Recall that $\operatorname{rank} R(G, p)=\operatorname{rank} R(G, p)_{v}=r_{d}(G)$ for all $v \in V$. For $F \subseteq D \cup L$ we will use $G_{F}$ to denote the mixed graph with vertex-set $V$ and labelled edge-set given by $F$. Note that $R\left(G_{F}, p\right)$ is the submatrix of $R(G, p)$ containing the rows indexed by $F$.

Proof of Lemma 1.2 Suppose $G=(V ; D, L)$ is a mixed graph, $v \in V_{0},(G, p)$ is a generic direction-length framework in $\mathbb{R}^{d}$, and $r_{d}(G)=d|V|-d-t$, for some $t \geq 1$. We need to prove that the framework space $S_{G, p, v_{0}}$ is a smooth manifold of dimension $t$. It will be convenient to construct this space in two stages using the pure frameworks $G_{D}$ and $G_{L}$. In the first stage, we 'factor out the direction constraints' by restricting to the framework space $S_{G_{D}, p, v_{0}}$, i.e. the set of all $q \in \mathbb{R}^{d|V|-d}$ such

\footnotetext{
${ }^{1}$ The usual definition for a semi-algebraic set uses polynomials with coefficients in $\mathbb{R}$, or more generally, in a real closed field. The fact that the Tarski-Seidenberg Theorem holds for semi-algebraic sets over $\mathbb{Q}$ follows from the original papers [10, 12].
} 
that $\left(G_{D}, q\right)$ is equivalent to $\left(G_{D}, p\right)$. Since direction constraints are linear, $S_{G_{D}, p, v_{0}}$ is a subspace of $\mathbb{R}^{d|V|-d}$ of codimension $r_{d}\left(G_{D}\right)$.

We let $p_{0}$ be the realisation obtained from $p$ by translating $v_{0}$ to the origin, and identify $p_{0}$ with a generic vector in $\mathbb{R}^{d|V|-d}$. Note that $R\left(G, p_{0}\right)=R(G, p)$. Define $F: \mathbb{R}^{d|V|-d} \rightarrow \mathbb{R}^{|L|}$ where for each $q \in \mathbb{R}^{d|V|-d}$ the coordinate of $F(q)$ corresponding to $e=u v \in L$ is $\|q(u)-q(v)\|^{2}$. Let $f$ be the restriction of $F$ to $S_{G_{D}, p, v_{0}}$. Then we can write the framework space for $(G, p)$ as $S_{G, p, v_{0}}=f^{-1}\left(f\left(p_{0}\right)\right)$. We will show that $f\left(p_{0}\right)$ is a regular value of $f$ and that rank $\left.d f\right|_{p_{0}}=r_{d}(G)-r_{d}\left(G_{D}\right)$. Then Lemma 3.2 will imply that $S_{G, p, v_{0}}$ is a smooth manifold of dimension $\operatorname{dim} S_{G_{D}, p, v_{0}}-$ $\left.\operatorname{rank} d f\right|_{p_{0}}=d|V|-d-r_{d}\left(G_{D}\right)-\left(r_{d}(G)-r_{d}\left(G_{D}\right)\right)=t$, as required.

We divide the argument into the following 5 steps:

Step 1 introduces a projection $h$ of $f$ and shows that $\left.\operatorname{rank} d f\right|_{p_{0}}=\left.\operatorname{rank} d h\right|_{p_{0}}=$ $r_{d}(G)-r_{d}\left(G_{D}\right)$.

Step 2 shows that $p_{0}$ is a regular point of both $f$ and $h$.

Step 3 defines a semi-algebraic set $K$ such that if $p_{0} \notin K$ then $h\left(p_{0}\right)$ is a regular value of $h$.

Step 4 shows that $p_{0} \notin K$.

Step 5 deduces that $f\left(p_{0}\right)$ is a regular value of $f$.

Step 1. To compute the rank of $d f$ at $p_{0}$ we apply Lemma 3.5 to $F$ and $f$, where $A=R\left(G_{D}, p\right)_{v_{0}}, X=S_{G_{D}, p, v_{0}}$ and $B=\left.d F\right|_{p_{0}}=2 R\left(G_{L}, p\right)_{v_{0}}$. Since multiplying rows by a constant does not change the rank, we get rank $\left.d f\right|_{p_{0}}=\operatorname{rank} R(G, p)_{v_{0}}-$ $\operatorname{rank} R\left(G_{D}, p\right)_{v_{0}}=r_{d}(G)-r_{d}\left(G_{D}\right)$. Also, we can choose $L^{\prime} \subseteq L$ so that $\left|L^{\prime}\right|=$ $r_{d}(G)-r_{d}\left(G_{D}\right)$, and writing $G^{\prime}=\left(V ; D, L^{\prime}\right)$ we have $r_{d}\left(G^{\prime}\right)=r_{d}(G)$. Let $H$ : $\mathbb{R}^{d|V|} \rightarrow \mathbb{R}^{\left|L^{\prime}\right|}$ be obtained from $F$ by projection onto the coordinates corresponding to edges in $L^{\prime}$. Let $h$ be the restriction of $H$ to $S_{G_{D}, p, v_{0}}$. We can similarly apply Lemma 3.5 to $H$ and $h$, where $A=R\left(G_{D}, p\right)_{v_{0}}, X=S_{G_{D}, p, v_{0}}$ and $B=\left.d H\right|_{p_{0}}=$ $2 R\left(G_{L^{\prime}}, p\right)_{v_{0}}$ to see that $\left.\operatorname{rank} d h\right|_{p_{0}}=\operatorname{rank} R\left(G^{\prime}, p\right)-\operatorname{rank} R\left(G_{D}, p\right)=r_{d}(G)-$ $r_{d}\left(G_{D}\right)=\left.\operatorname{rank} d f\right|_{p_{0}}$.

Step 2. Next we claim that $p_{0}$ is a regular point of both $f$ and $h$. We need to show that $\left.\operatorname{rank} d f\right|_{q} \leq\left.\operatorname{rank} d f\right|_{p_{0}}$ and rank $\left.d h\right|_{q} \leq\left.\operatorname{rank} d h\right|_{p_{0}}$ for any $q \in S_{G_{D}, p, v_{0}}$. Say that $q$ is degenerate if $q(u)=q(v)$ for some $u v \in D$, otherwise $q$ is non-degenerate. Suppose first that $q$ is non-degenerate. Then each row of $R\left(G_{D}, q\right)$ is a non-zero multiple of the corresponding row in $R\left(G_{D}, p\right)$, so $\operatorname{rank} R\left(G_{D}, q\right)_{v_{0}}=\operatorname{rank} R\left(G_{D}, p\right)_{v_{0}}$. We also have $\operatorname{rank} R(G, q)_{v_{0}} \leq \operatorname{rank} R(G, p)_{v_{0}}$ as $p$ is generic, so $\left.\operatorname{rank} d f\right|_{q}=$ rank $R(G, q)_{v_{0}}-\operatorname{rank} R\left(G_{D}, q\right)_{v_{0}} \leq\left.\operatorname{rank} d f\right|_{p_{0}}$. On the other hand, if $q$ is degenerate we consider a sequence of points $\left(q_{i}\right)_{i \geq 1} \in S_{G_{D}, p, v_{0}}$ such that each $q_{i}$ is nondegenerate and $\left(q_{i}\right)$ converges to $q$. Then rank $\left.d f\right|_{q_{i}} \leq\left.\operatorname{rank} d f\right|_{p_{0}}$ for all $i \geq 1$, and taking the limit we obtain $\left.\operatorname{rank} d f\right|_{q} \leq\left.\operatorname{rank} d f\right|_{p_{0}}$. The same argument applies with $h$ instead of $f$.

Step 3. Now we will reformulate our definitions using semi-algebraic sets. Let $x=$ $\left(x_{1}, \ldots, x_{d|V|-d}\right)$ and $y=\left(y_{1}, \ldots, y_{d|V|-d}\right)$ be vectors of indeterminates, which we think of as representing standard position frameworks. We define polynomials $P_{e}(x, y)=H(x)_{e}-H(y)_{e}$ for $e \in L^{\prime}$ and $P_{i}(x, y)=R\left(G_{D}, x\right)_{i} \cdot y$ for each row $R\left(G_{D}, x\right)_{i}$ of $R\left(G_{D}, x\right)$. We also let $M\left(G^{\prime} ; x, y\right)$ be the matrix $\left(\begin{array}{c}R\left(G_{D}, x\right) \\ R\left(G_{L^{\prime}}, y\right)\end{array}\right)$, and for each $r_{d}(G) \times r_{d}(G)$ minor $N$ we define $P_{N}(x, y)=\operatorname{det} M\left(G^{\prime} ; x, y\right)[N]$. 
Then we let $K_{0} \subseteq \mathbb{R}^{2 d|V|-2 d}$ be the (semi-)algebraic set defined by setting all of these polynomials to equal zero and let $K=\left\{x: \exists y,(x, y) \in K_{0}\right\}$ be the projection of $K_{0}$ onto the first $d|V|-d$ coordinates. Note that $(x, y) \in K_{0}$ if and only if $H(x)=H(y), y \in S_{G_{D}, x, v_{0}}$ and $\operatorname{rank} M\left(G^{\prime} ; x, y\right)<r_{d}(G)$. Also, Lemma 3.5 implies that $\left.\operatorname{rank} d h\right|_{y}=\operatorname{rank} M\left(G^{\prime} ; p_{0}, y\right)-r_{d}\left(G_{D}\right)$ for any $y \in S_{G_{D}, p, v_{0}}$. Now if $h\left(p_{0}\right)$ were not a regular value of $h$, then there would be some $y \in S_{G_{D}, p, v_{0}}$ with $H(y)=H\left(p_{0}\right)$ and $\left.\operatorname{rank} d h\right|_{y}<\left.\operatorname{rank} d h\right|_{p_{0}}$. This would give $\operatorname{rank} M\left(G^{\prime} ; p_{0}, y\right)=$ $\left.\operatorname{rank} d h\right|_{y}+r_{d}\left(G_{D}\right)<\left.\operatorname{rank} d h\right|_{p_{0}}+r_{d}\left(G_{D}\right)=r_{d}(G)$, and so $p_{0} \in K$. It therefore suffices to prove that $p_{0} \notin K$.

Step 4. Suppose for a contradiction that $p_{0} \in K$. By Theorem 5.1, $K$ is semialgebraic over $\mathbb{Q}$. Since $p$ is generic we have $Q\left(p_{0}\right) \neq 0$ for all $Q \in \mathbb{Q}\left[X_{1}, \ldots\right.$, $\left.X_{d|V|-d}\right]$, so we can write $K=\left\{x \in \mathbb{R}^{m}: Q_{i}(x)>0,1 \leq i \leq t\right\}$ for some $Q_{1}, \ldots, Q_{t} \in \mathbb{Q}\left[X_{1}, \ldots, X_{d|V|-d}\right]$. Then by continuity some open neighbourhood $U$ of $p_{0}$ in $\mathbb{R}^{d|V|-d}$ is contained in $K$. Let $T$ be the set of all points $q \in \mathbb{R}^{d|V|-d}$ such that $(G, q)$ has no vertical direction edges. Note that $p_{0} \in T$, since $p$ is generic. Also, $T$ is open in $\mathbb{R}^{d|V|-d}$, so we can assume that $U \subseteq T$. Let $W=U \cap S_{G_{D}, x, v_{0}}$. Then $W$ is an open neighbourhood of $p_{0}$ in $S_{G_{D}, x, v_{0}}$.

We claim that $h(x)$ is a critical value of $h$ for every $x \in W$. For consider any $x \in W$. Then $x \in T$ is non-degenerate, so each row of $R\left(G_{D}, x\right)$ is a nonzero multiple of the corresponding row in $R\left(G_{D}, p\right)$. Also $x \in K$, so there is some $y \in S_{G_{D}, p, v_{0}}$ with $h(x)=h(y)$ and $\operatorname{rank} M\left(G^{\prime} ; x, y\right)<r_{d}(G)$. Repeating the calculation above, we see that $\left.\operatorname{rank} d h\right|_{y}=\operatorname{rank} M\left(G^{\prime} ; p_{0}, y\right)-r_{d}\left(G_{D}\right)=$ $\operatorname{rank} M\left(G^{\prime} ; x, y\right)-r_{d}\left(G_{D}\right)<r_{d}(G)-r_{d}\left(G_{D}\right)$, which proves the claim.

Since $p_{0}$ is a regular point of $h$, Lemma 3.3 implies that we can choose an open neighbourhood $W^{\prime} \subseteq W$ of $p_{0}$ in $S_{G_{D}, x, v_{0}}$ such that $h$ maps $W^{\prime}$ diffeomorphically onto $h\left(W^{\prime}\right)$. In particular, it follows that the set of critical values of $h$ has non-zero measure, which is in contradiction to Sard's Theorem (Theorem 3.1). We deduce that $p_{0} \notin K$.

Step 5. By Steps 3 and 4 , it follows that $h\left(p_{0}\right)$ is a regular value of $h$. It follows that $f\left(p_{0}\right)$ is a regular value of $f$, as $f^{-1}\left(f\left(p_{0}\right)\right) \subseteq h^{-1}\left(h\left(p_{0}\right)\right)$, so for every $x \in f^{-1}\left(f\left(p_{0}\right)\right)$ we have $\left.\operatorname{rank} d f\right|_{x} \geq\left.\operatorname{rank} d h\right|_{p_{0}}=\left.\operatorname{rank} d f\right|_{p_{0}}$. Since rank $\left.d f\right|_{p_{0}}=$ $r_{d}(G)-r_{d}\left(G_{D}\right)$ by Step 1, Lemma 3.2 implies that $S_{G, p, v_{0}}=f^{-1}\left(f\left(p_{0}\right)\right)$ is a smooth manifold of dimension $t$, as required.

\section{Concluding Remarks}

Theorem 1.1 would become false if we removed the hypothesis that the mixed graph has at least two length edges. This can be seen by considering a mixed graph $G=$ $(V ; D, L)$ which is rigid in $\mathbb{R}^{d}$ and has a unique length edge $e$. Let $(G, p)$ be a generic realisation of $G$ in $\mathbb{R}^{d}$ and $(G, q)$ be an equivalent realisation. Then $(G \backslash e, p)$ is not rigid since it has only direction edges so admits an arbitrary dilation. On the other hand, $r_{d}(G \backslash e, p)=d|V|-d-1$ and so $(G \backslash e, p)$ is direction globally rigid by results of Whiteley [13]. Thus $(G, q)$ can be obtained from $(G, p)$ by a translation and a dilation. The existence of the length edge $e$ now implies that the only possible dilations are by \pm 1 . Hence $(G, q)$ is congruent to $(G, p)$ and $(G, p)$ is globally rigid. 

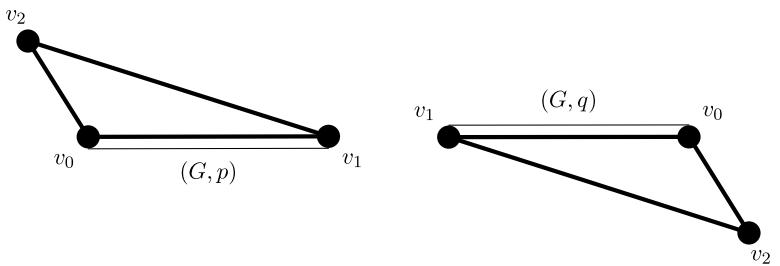

Fig. 2 Let $e$ be the direction edge joining $v_{0}$ and $v_{1}$. Then the unique motion of $(G \backslash e, p)$ which keeps $v_{0}$ fixed is a rotation about the point $p\left(v_{0}\right)$. It follows that the connected component of the framework space $S_{G \backslash e, p, v_{0}}$ which contains $p$ has a unique point $q \neq p$ such that $(G, q)$ is equivalent to $(G, p)$. Since $(G, q)$ can be obtained from $(G, p)$ by a dilation by -1 through the point $p\left(v_{0}\right),(G, q)$ is congruent to $(G, p)$. Note that the framework $(G, p)$ is not a counterexample to Conjecture 6.1 since it is not globally rigid: we can obtain an equivalent but non-congruent framework by reflecting $(G, p)$ in the line through $p\left(v_{0}\right)$ and $p\left(v_{1}\right)$. The problem is that we cannot reach this realisation by a continuous motion of $(G \backslash e, p)$

Theorem 1.3 would become false if we removed the possibility that $G \backslash e$ is unbounded in $\mathbb{R}^{2}$. This can be seen by considering a mixed graph $G=(V ; D, L)$ in which all pairs of vertices of $V \backslash\{v\}$ are joined by both a direction and length edge and $v$ is joined to two distinct vertices $x, y$ by two direction edges $e=v x, f=v y$. It is easy to see that for any generic realisation $(G, p)$ of $G$ in $\mathbb{R}^{2},(G, p)$ is globally rigid and $(G \backslash e, p)$ is unbounded.

We believe that the hypothesis of Theorem 1.3 that ' $G \backslash e$ has a rigid subgraph with more than one vertex' may not be necessary for mixed graphs with at least two length edges.

Conjecture 6.1 [7] Suppose $(G, p)$ is a 2-dimensional generic globally rigid direction-length framework with at least two length edges and e is a direction edge of $G$. Then $G \backslash e$ is either rigid or unbounded in $\mathbb{R}^{2}$.

The framework in Fig. 2 illustrates the difficulty in using the proof technique of Theorem 1.3 to verify this conjecture.

Acknowledgements We would like to thank Bob Connelly and Tibor Jordán for helpful discussions. We are particularly indebted to Bob for his suggestion to 'factor out by the direction constraints' in the proof of the Manifold Lemma. Furthermore, we thank an anonymous referee for several insightful comments. These included an improvement to the proof of Theorem 1.1, which we have implemented. They also included a possible alternative proof of Lemma 1.2 based on the algebraic form of Sard's theorem. We have not explored this option, as the method of our current proof seems to also be interesting, and we do not want to use algebraic geometry where more elementary means are available.

\section{References}

1. Connelly, R.: Generic global rigidity. Discrete Comput. Geom. 33, 549-563 (2005)

2. Gortler, S., Healy, A., Thurston, D.: Characterizing generic global rigidity (2007). arXiv:0710.0926

3. Hendrickson, B.: Conditions for unique graph realizations. SIAM J. Comput. 21, 65-84 (1992)

4. Jackson, B., Jordán, T.: Connected rigidity matroids and unique realizations of graphs. J. Comb. Theory, Ser. B 94, 1-29 (2005)

5. Jackson, B., Jordán, T.: Globally rigid circuits of the direction-length rigidity matroid. J. Comb. Theory, Ser. B 100, 1-23 (2010) 
6. Jackson, B., Jordán, T.: Operations preserving global rigidity of generic direction-length frameworks. Int. J. Comput. Geom. Appl. 20, 685-708 (2010)

7. Jackson, B., Keevash, P.: Bounded direction-length frameworks. Discrete Comput. Geom. (2011). doi:10.1007/s00454-011-9325-0

8. Lee, J.M.: Introduction to Smooth Manifolds. Springer, Berlin (2003)

9. Milnor, J.W.: Topology from the Differentiable Viewpoint. University Press of Virginia, Charlottesville (1965)

10. Seidenberg, A.: A new decision method for elementary algebra. Ann. Math. 60, 365-374 (1954)

11. Servatius, B., Whiteley, W.: Constraining plane configurations in CAD: combinatorics of directions and lengths. SIAM J. Discrete Math. 12, 136-153 (1999)

12. Tarski, A.: A decision method for elementary algebra and geometry. Manuscript. Santa Monica, CA: RAND Corp. (1948). Republished as A Decision Method for Elementary Algebra and Geometry, 2nd edn. University of California Press, Berkeley, CA (1951)

13. Whiteley, W.: Some matroids from discrete applied geometry. In: Matroid Theory. AMS Contemporary Mathematics, vol. 197, pp. 171-313 (1996) 